\title{
A Literature Review to Assess Blood Loss in Minimally Invasive Liver Surgery Versus in Open Liver Resection
}

\author{
Eiad Elmahi ${ }^{1}$, Yahya Salama ${ }^{2}$, Fergal Cadden ${ }^{1}$ \\ 1. General Surgery, Lincoln County Hospital, Lincoln, GBR 2. Surgery, Kettering General Hospital, Kettering, GBR
}

Corresponding author: Eiad Elmahi, eiad-elmahi@hotmail.co.uk

\section{Abstract}

\section{Aim and objectives}

The aim of the study was to assess the amount of blood loss in minimally invasive hepatectomy and open liver resection for both benign and neoplastic conditions.

\section{Introduction}

Minimally invasive surgery has progressively developed to a stage where once-novel and highly specialized surgical techniques are now common practice. Colorectal surgery is the key example that has shown minimally invasive surgery as highly beneficial. Successes in the colorectal laparoscopic approach have now been integrated into the speciality of hepatopancreaticobiiary (HPB) surgery. In this review, we will compare the amount of blood loss in minimally invasive liver resection with the more traditional approach of open liver resection.

\section{Methods}

A literature review was conducted which included the length of patient mobilization as a postoperative complication following laparoscopic and open liver resections. Medline, PubMed, and Cochrane were accessed to review previously published studies. Twelve studies were selected, and all of them were in English, ranged from the year 2000 to 2020.

\section{Results}

Eleven out of the 12 included studies indicated that minimally invasive liver resection is associated with reduced blood loss.

\section{Conclusion}

In comparing both minimally invasive liver resection and classic open surgery, minimally invasive liver resection was shown to have reduced blood loss; this was seen in both malignant and benign tumours. Therefore, laparoscopic liver resection could be favoured over the classical open approach to avoid excessive

Review began 05/27/2021 Review ended 06/28/2021 Published 06/28/2021

\section{(c) Copyright 2021}

Elmahi et al. This is an open access article distributed under the terms of the Creative Commons Attribution License CC-BY 4.0., which permits unrestricted use, distribution, and reproduction in any medium, provided the original author and source are credited.
Categories: General Surgery

Keywords: "laparoscopic hepatectomy”, "post-operative complications”, "post-hepatectomy bleeding”, "intraoperative bleeding”, "open liver resection”, "hepatocellular carcinoma”, "benign liver tumours”, "minimally invasive", "hepatobiliary surgery", "segmentectomy”. the term and "versus" was used to identify studies which compared the outcomes of both techniques

\section{Introduction}

The introduction of the laparoscopic approach to many surgical practices has greatly altered the standard practice of many surgical specialities, through repeated reviews in the literature, it has proven to be superior in terms of postoperative outcomes for patients. It has seen a slow adaptation to HPB surgery in general where an open approach is opted for more frequently. The laparoscopic approach is less commonly applied for liver resection. This has been a point of contention as there are few studies to objectively compare the outcomes of the laparoscopic technique to the open technique. However, with the few studies that directly compare both practices, there are indications that a laparoscopic approach show reduced rates of postoperative complications [1].

In this study, we attempt to collate the available evidence for laparoscopic liver resection (LLR) and show evidence that there are benefits to the practice of laparoscopic liver resection compared to open liver resection (OLR). Comparing the evidence on minimally invasive and open techniques in liver resection will provide further insight into the premise that the laparoscopic approach reduced the amount of blood loss. 
Postoperative hospital stay is a longstanding issue regarding a patient's recovery; prolonged hospital stay is associated with an increased risk of developing hospital-acquired infections and at a greater cost to the hospital [2]. By reviewing the available evidence, we hope to see that minimally invasive surgery helps facilitate reduced postoperative hospital stay. If the evidence suggests that the laparoscopic approach reduces the amount of blood loss intra-operatively, minimally invasive surgery may be recommended for liver resection over the traditional approach of open liver resection [3].

Postoperative bleeding using both techniques will be weighed. The type of liver resection and the age group (45-70 years) will be considered in this review to avoid complications not related to hepatectomies (cardiorespiratory, anaesthesia-related complications) [4].

Liver tumours are classified as benign or malignant, they are not uncommon. The most common benign liver tumour is haemangioma, which mainly affects the right lobe of the liver. In general, haemangiomas are usually asymptomatic and are usually associated with certain conditions like Klippel-Trenaunary syndrome. These are usually found as incidental findings during imaging (ultrasound scan and CT) [5]. The indications for liver resections in these cases include right upper abdominal fullness, bleeding, hemorrhagic shock, and gastric outlet obstruction [6]. Other types of benign tumours are hepatic adenoma which is more frequently associated with females on the oral contraceptive pill [7]. Rare benign tumours include cystadenoma, lipoma, fibroma, and leiomyoma which are symptomatic unless they cause complications [5].

Malignant tumours of the liver are the sixth most common cause of death globally and the third cause of mortality. Liver malignancy is more common in Asia and Central Africa; this could be attributed to aflatoxincontaminated peanuts intake which is associated with a higher incidence of developing primary liver malignancy [8].

In terms of liver malignancy, they are categorized into primary or secondary. Primary liver malignancy is either a hepatoma or hepatocellular carcinoma (HCC). A secondary malignancy is caused by liver metastasis. Risk factors for HCC are varied, including high BMI, male gender, liver cirrhosis, high alcohol consumption, aflatoxin-contaminated food, and hepatitis [8].

Liver metastasis is the most common indication for liver resection, and colorectal cancer is the commonest primary source [9]. Hepatectomy is offered to patients with localized disease; unfortunately, approximately half of the patients develop disease recurrence [9]. The reasoning behind liver resection in metastatic colorectal disease is that there is a potential for a curative outcome and a better prognosis with surgical intervention than conservative treatment [8].

The use of laparoscopic liver resection is an umbrella term to describe different techniques of liver resection. These techniques include pure laparoscopy, hand-assisted laparoscopy, and laparoscopy-assisted methods [10]; these subtypes will also be included in this review.

Liver resection is considered a major operation. Its complications of course will need to be anticipated as postoperative complications are likely to develop in both anatomical and non-anatomical liver resections. Complications include bleeding, infection, and injury to surrounding structures, bile leak, and increasing length of postoperative stay. As expected, these reflect increased risk in morbidity and mortality as well as a prolonged hospital stay [11].

\section{Materials And Methods}

\section{Search strategy}

A literature review was conducted, to compare post liver resection bleeding in both laparoscopic and open techniques. This search had adhered to the PRISMA (Preferred Reporting Items for Systematic Reviews and Meta-Analyses) (Figure 1). We aimed to identify appropriate comparative studies and clinical trials. The period of review ranged from 2000 to 2020. PubMed, Medline, Cochrane, and Google Scholar were searched to find relevant studies. 


\section{Cureus}

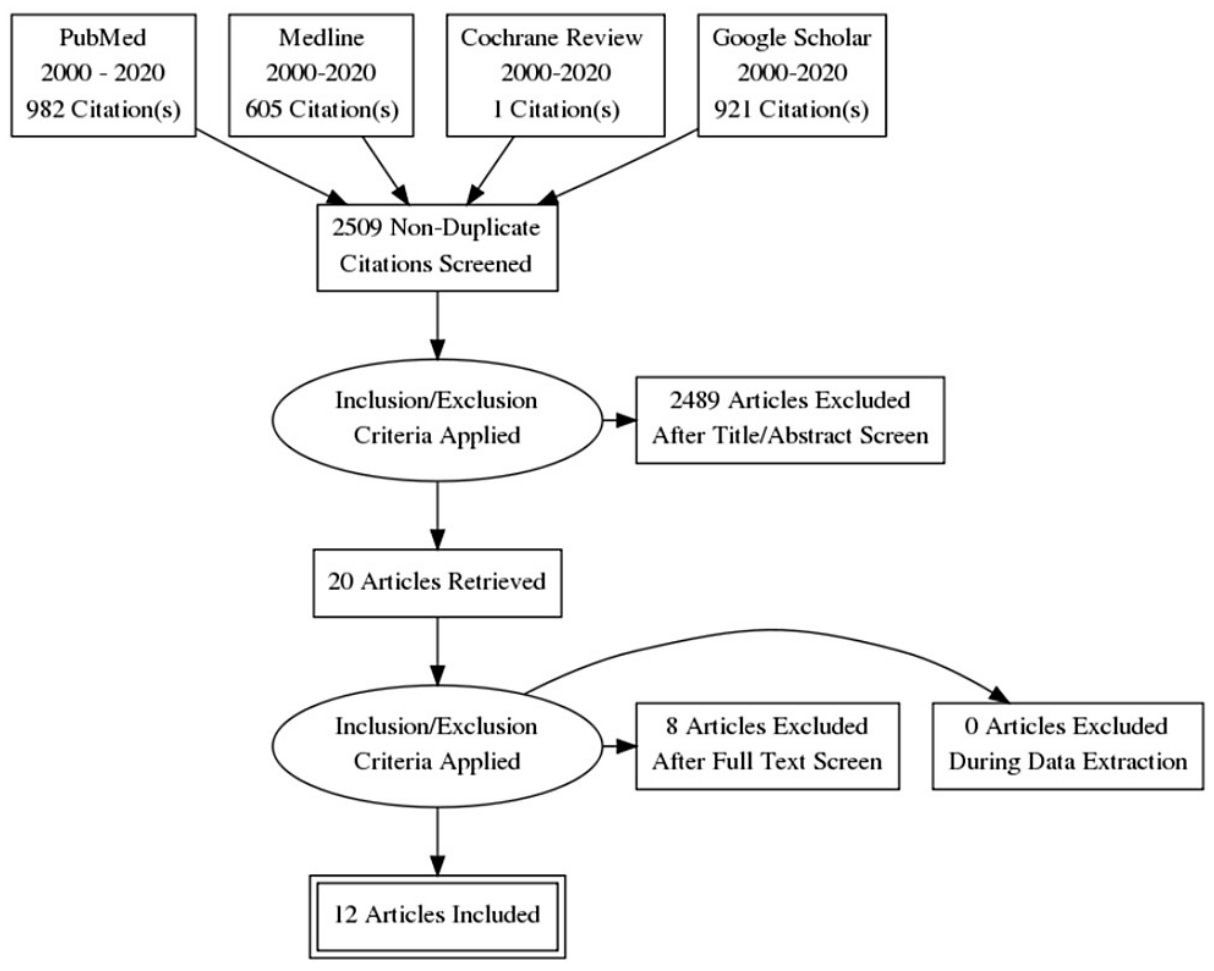

FIGURE 1: PRISMA Diagram

PRISMA: Preferred Reporting Items for Systematic Reviews and Meta-Analyses

Keywords included "laparoscopic hepatectomy”, "Post-operative complications”, "post-hepatectomy bleeding”, "intra-operative bleeding”, “open liver resection”, "hepatocellular carcinoma”, "benign liver tumors”, "minimally invasive”, "hepatobiliary surgery”, “segmentectomy”. The term AND "versus” was used to identify studies that compared the outcomes of both techniques.

\section{Research protocol}

Studies comparing the short-term complication of postoperative blood loss in both minimally invasive and traditional open technique liver resection were subject to inclusion and exclusion criteria. The identification, quality assessment, data extraction, and statistical analyses of the studies will be included in the research protocol.

Published papers and studies were searched using the electronic databases described above. The PICO (population, intervention, control, and outcomes) format was also used to help select the most relevant studies that provide a balanced view of the outcomes. Published studies and trials during the period ranging from 2000 to 2020 were included in the review.

\section{Inclusion criteria}

All quantitative studies from 2000 to 2020 meeting the following criteria were reviewed in this systematic review: studies and publications highlighting and comparing the postoperative complication of blood loss in LLR and OLR, the indication for each surgery, type of procedure, specific postoperative complications related directly to both techniques, and, most importantly, comparing the degree of blood loss in both techniques. All studies included in this review were published in English and describe a similar patient population.

\section{Exclusion criteria}

The exclusion criteria used for this review included the following: no reported outcome allowing for quantifiable comparison, liver resection performed due to metastatic liver disease, studies not published in English, and unable to extract data from results.

\section{Study selection and quality assessment}

The Effective Public Health Practice Project (EPHPP) quality assessment tool was used for this review to adequately assess the quality of each publication. It assesses the trustworthiness of each study and ensures 


\section{Cureus}

the efficiency and reliability of the information included in the publications analyzed. It is a tool that was initially used in the practice of public health but has been proven to be effective in other research fields.

\section{Data extraction and analysis}

A data extraction form is utilized to collect the relevant information to address the question set out in this systematic review. The World Health Organization guidelines were also used to assess the complications that can occur after liver resections using a laparoscopic and open technique. Postoperative blood loss is a common complication of surgery and is kept at a minimum as much as possible as bleeding negatively affects postoperative recovery significantly [3].

\section{Results}

\section{Literature search}

A thorough literature search identified 2509 publications using keywords: 982 in PubMed, 605 in Medline, 921 in Google Scholar, and one in Cochrane Reviews (Table 1). Of the 2509 publications, 2489 had been excluded due to non-relevant or non-comparative studies; 20 satisfied the inclusion criteria; 8 were excluded for duplication; and 12 were included in this systematic review. We did not identify any randomized controlled trials (RCTs) that compared the outcomes of LLR and OLR in our literature review.

PubMed search, Accessed on
November 20th, 2020 (921 Articles)
(laparoscopic hepatectomy
OR Minimally invasive) AND (Post-
operative complications OR Post-
hepatectomy bleeding OR Intra-operative
bleeding) AND (Open liver resection)
AND (Versus AND Compare) OR
(Hepatobiliary surgery AND
Segmentectomy)

Medline search, Accessed

on December 20th, 2020 (605 Articles)

(laparoscopic hepatectomy OR Minimally invasive) AND (Postoperative complications OR Post-

hepatectomy bleeding OR Intraoperative bleeding) AND (Open liver resection) AND (Versus AND Compare) OR (Hepatobiliary surgery AND Segmentectomy)
Cochrane Reviews search, Accessed on November 20th, 2020 (1 Article)

(laparoscopic hepatectomy OR Minimally invasive) AND (Post-operative complications OR Posthepatectomy bleeding $\mathrm{OR}$ Intra-operative bleeding) AND (Versus AND Compare)
Google Scholar search, Accessed on December 20th, 2020 (921 Articles)

(laparoscopic hepatectomy OR Minimally invasive) AND (Postoperative complications OR Posthepatectomy bleeding OR Intraoperative bleeding) AND (Open liver resection) AND (Versus AND Compare) OR (Hepatobiliary surgery AND Segmentectomy)

\section{TABLE 1: The Search Syntax}

\section{Demographics}

A total of 12 studies were included in the review, all retrospective in design, which included a total of 287 OLRs and 480 LLRs. The mean blood loss in LLR was $179.1 \mathrm{~mL}$ and in OLR was $295.64 \mathrm{~mL}$. The mean age for OLR was 55.4 years and for LLR was 56.7 years (Table 2) 


\section{Cureus}

Characteristics and demographics

Studies included in the systematic review 12

Retrospective vs Prospective

All Retrospective

Total Number of Open Resections

Total Number of laparoscopic liver resections

480

Mean Blood Loss LLR

$179.10 \mathrm{ml}$

Mean Blood Loss OLR

$295.64 \mathrm{ml}$

Mean Age (years) in OLR

55.4

Mean Age (years) in LLR

56.7

Mortality ( $<30$ days) in OLR

Mortality (<30 days) in LLR

0

TABLE 2: Patient Demographics

LLR: laparoscopic liver resection; OLR: open liver resection

Comparing rates of blood loss of LLR and OLR

The postoperative parameter of blood loss[3]was used to compare the outcomes of LLR and OLR (Table 3). Lesurtel, et al[12],had compared 18 LLR to 20 OLR which had highlighted less blood loss for laparoscopic liver resection at $288 \mathrm{~mL}$ compared to $485 \mathrm{~mL}$ for the OLR group. Buell, et al[13]compared the outcome of 17 LLR to 100 OLR for benign tumours. They identified that the mean blood loss for LLR was $299 \mathrm{~mL}$ compared to $485 \mathrm{~mL}$ for the OLR group (P0.05). Morino, et al[14] enrolled 60 patients for a comparative study, 30 LLR and 30 OLR. In the LLR group, blood loss was $320 \mathrm{~mL}$ compared to $479 \mathrm{~mL}$ in OLR ( $\mathrm{P} \leqslant 0.05)$. Koffron, et al[15]evaluated 300 liver resections, 241 LLR and 59 hybrid cases (OLR and laparoscopic-assisted). They identified that blood loss for LLR was $102 \mathrm{~mL}$ compared to $325 \mathrm{~mL}$ loss in the hybrid group. Aldrighetti, et al[16]compared 20 LLR to 20 OLRwhich showed thatblood loss for LLR and OLR was $165 \mathrm{~mL}$ and $214 \mathrm{~mL}$, respectively ( $\mathrm{P}=0.001$ ). Polignano, et al[17]included 50 patients, 25 LLR and $25 \mathrm{OLR}$, which showed a reduced volume of blood loss in LLR compared to OLR ( $\mathrm{P} \leqslant 0.003)$. Slakey, et al[18]reviewed 62 patients, 45 LLR and 17 OLR, which showed that hospital blood loss was significantly less in the LLR group compared to OLR $(P \leqslant 0.0001)$. Qiu, et al[19]compared 49 patients, 24 LLR and 25 OLR, showing a reduced blood loss in the LLR group ( $210 \mathrm{~mL}$ in LLR, 380mL in OLR) ( $\mathrm{P} \leqslant 0.01$ ). Zhang, et al[ 1$]$ had evaluated 50 patients (30 LLR to 20 OLR) which indicated reduced blood loss in LLR compared to OLR ( $200 \mathrm{~mL}$ vs $328 \mathrm{~mL})(\mathrm{P}<0.05)$. Nassar, et al [20]also identified reduced blood loss in the LLR group compared to OLR $(\mathrm{P} \leqslant 0.001)$. However, one study completed by Lau, et al[21]indicated that there was no statistical difference related to blood loss (LLR $386 \mathrm{~mL}$, OLR 556mL, $\mathrm{P}=0.216$ ). Hu, et al [22] also identified no statistical difference when comparing blood loss, 30 OLR and 30 LLR patients were selected for the study $(\mathrm{P} \geqslant 0.05)$. 


\begin{tabular}{|c|c|c|c|c|c|c|c|c|}
\hline $\begin{array}{l}\text { Study } \\
\text { Author(s) }\end{array}$ & Year & Location & Study type & $\begin{array}{l}\text { Numbers of } \\
\text { patients }\end{array}$ & LLR/OLR & $\begin{array}{l}\text { Eligibility for } \\
\text { inclusion }\end{array}$ & $\begin{array}{l}\text { Quality } \\
\text { assessment }\end{array}$ & $\begin{array}{l}\text { Ethical } \\
\text { appraisal }\end{array}$ \\
\hline Lesurtel et al & 2003 & France & $\begin{array}{l}\text { Comparative } \\
\text { matched }\end{array}$ & 38 & $18 / 20$ & Yes & Yes & Yes \\
\hline Morino et al & 2003 & Italy & $\begin{array}{l}\text { Comparative } \\
\text { matched }\end{array}$ & 60 & $30 / 30$ & Yes & Yes & Yes \\
\hline Buell et al & 2004 & USA & $\begin{array}{l}\text { Comparative } \\
\text { matched }\end{array}$ & 117 & $17 / 100$ & Yes & Yes & Yes \\
\hline Koffron et al & 2007 & USA & $\begin{array}{l}\text { Comparative } \\
\text { matched }\end{array}$ & 300 & $241 / 59$ & Yes & Yes & Yes \\
\hline $\begin{array}{l}\text { Aldrighetti et } \\
\text { al }\end{array}$ & 2008 & Italy & $\begin{array}{l}\text { Comparative } \\
\text { matched }\end{array}$ & 40 & $20 / 20$ & Yes & Yes & Yes \\
\hline $\begin{array}{l}\text { Polignano et } \\
\text { al }\end{array}$ & 2008 & UK & $\begin{array}{l}\text { Comparative } \\
\text { matched }\end{array}$ & 50 & $25 / 25$ & Yes & Yes & Yes \\
\hline Hu et al & 2011 & China & $\begin{array}{l}\text { Comparative } \\
\text { matched }\end{array}$ & 60 & $30 / 30$ & Yes & Yes & Yes \\
\hline Slakey et al & 2013 & USA & $\begin{array}{l}\text { Retrospective } \\
\text { review }\end{array}$ & 62 & $45 / 17$ & Yes & Yes & Yes \\
\hline Qiu, et al & 2014 & USA & $\begin{array}{l}\text { Comparative } \\
\text { matched }\end{array}$ & 49 & $24 / 25$ & Yes & Yes & Yes \\
\hline Lau et al & 2015 & USA & $\begin{array}{l}\text { Case matched } \\
\text { controlled }\end{array}$ & 125 & $47 / 78$ & Yes & Yes & Yes \\
\hline Nassar et al & 2015 & Egypt & $\begin{array}{l}\text { Comparative } \\
\text { matched }\end{array}$ & 30 & $15 / 15$ & Yes & Yes & Yes \\
\hline Zhang et al & 2015 & China & Cohort study & 50 & $30 / 20$ & Yes & Yes & Yes \\
\hline
\end{tabular}

\section{TABLE 3: Selected Studies Comparing the Rates of Blood Loss Of LLR and OLR}

LLR: laparoscopic liver resection; OLR: open liver resection

\section{Discussion}

Published reviews on laparoscopic liver resection describe technically challenging cases (peripherally sited tumours, etc.) that may discourage minimally invasive liver resection when compared to the open technique[23]. However, dueto the advances in laparoscopic approach over the past three decades, and the added benefit of a perceived reduction in postoperative complications, Koffron, et al[15], have advocated for laparoscopic liver resection to be the gold standard treatment for most hepatectomies.

Comparing OLR to LLR has been a controversial issue due to the lack of sound evidence, indicating the superiority of LLR to OLR, according to Laurent, et al[24]. Our aim is to compare the postoperative blood loss of both techniques. Till present, there is have been no published randomized controlled trials directly comparing both techniques. Because of the lack of high-quality evidence, it is more difficult to compare both approaches. However, the papers reviewed provide a strong indication that minimally invasive liver resection has reduced intraoperative and postoperative bleeding compared to open liver resection.

As discussed previously, one study suggested that there is no statistical difference between LLR and OLR in terms of blood loss. This may influence surgeons to opt for open liver resection techniques by comparison to laparoscopic liver resections as per studies by Laurent, et al [24] and Lau, et al [21]. It also reinforces the need for high-quality studies to provide more objective evidence in deciding the appropriate technique to opt for. The technical difficulty in performing laparoscopic liver resection and the demand for further specialized training is another argument against the use of minimally invasive liver resection. Technical issues such as a narrow operative field and the rigidity of minimally invasive instruments may concern surgeons and may have the perception of a higher risk of undesirable complications [23]. Location of tumour sites is another added concern as peripherally sited tumours may prove too complex for resection laparoscopically as seen in the study by Gagner, et al [25]. 
The study groups that have supported the benefit of LLR comparing OLR in terms of the amount of blood loss also recognize the issue of advanced laparoscopic technique for LLR but advocate the need for continuous development of laparoscopic techniques in light of the results of reduced postoperative complications in general [15].

\section{Conclusions}

Based on the literature available, LLR has a reduced amount of postoperative blood loss, it is an established and safe technique with appropriate training. As discussed previously, higher quality evidence such as randomized controlled trials would provide further insight into the comparative benefits of LLR. This reviewalso only considers the short-term postoperative issue. The blood lost during these studies were measured intra-operatively and the patients' blood loss post-procedure. In conclusion, this review shows there is a reduced intra-operative blood loss and provides a reasonable argument for the recommendation of LLR for patients undergoing hepatectomies both for benign and malignant conditions. It also provides a good case for the endorsement of further training in laparoscopic techniques related to liver resection, but as discussed, this requires higher quality studies such as RCTs to bolster the argument for LLR.

\section{Additional Information \\ Disclosures}

Human subjects: All authors have confirmed that this study did not involve human participants or tissue. Animal subjects: All authors have confirmed that this study did not involve animal subjects or tissue. Conflicts of interest: In compliance with the ICMJE uniform disclosure form, all authors declare the following: Payment/services info: All authors have declared that no financial support was received from any organization for the submitted work. Financial relationships: All authors have declared that they have no financial relationships at present or within the previous three years with any organizations that might have an interest in the submitted work. Other relationships: All authors have declared that there are no other relationships or activities that could appear to have influenced the submitted work.

\section{References}

1. Zhang Y, Chen XM, Sun DL: Comparison of laparoscopic versus open left lateral segmentectomy . Int J Clin Exp Med. 2015, 8:904-9.

2. Wang LH, Xu DJ, Wei XJ, Chang HT, Xu GH: Electrolyte disorders and aging: risk factors for delirium in patients undergoing orthopedic surgeries. BMC Psychiatry. 2016, 16:418. 10.1186/s12888-016-1130-0

3. Topal B, Fieuws S, Aerts R, Vandeweyer H, Penninckx F: Laparoscopic versus open liver resection of hepatic neoplasms: comparative analysis of short-term results. Surg Endosc. 2008, 22:2208-13. 10.1007/s00464008-0023-9

4. Sieber F, Barnett SR: Preventing postoperative complications in the elderly . Anesthesiol Clin. 2011, 29:8397.

5. Navarro AP, Gomez D, Lamb CM, Brooks A, Cameron IC: Focal nodular hyperplasia: a review of current indications for and outcomes of hepatic resection. HPB (Oxford). 2014, 16:503-11. 10.1111/hpb.12169

6. Benign liver tumours. (2020). Accessed: 10 Aug 2020: https://patient.info/doctor/benign-liver-tumours.

7. Hepatic adenoma. (2017). Accessed: 6 August 2020: https://radiopaedia.org/articles/hepatic-adenoma.

8. Herszényi L, Tulassay Z: Epidemiology of gastrointestinal and liver tumors. Eur Rev Med Pharmacol Sci. 2010, 14:249-58.

9. Siegel R, Desantis C, Jemal A: Colorectal cancer statistics, 2014. CA Cancer J Clin. 2014, 64:104-17. 10.3322/caac. 21220

10. Coelho FF, Kruger JA, Fonseca GM, et al.: Laparoscopic liver resection: experience based guidelines. World J Gastrointest Surg. 2016, 8:5-26. 10.4240/wjgs.v8.i1.5

11. Winslow ER, Brunt LM: Perioperative outcomes of laparoscopic versus open splenectomy: a meta-analysis with an emphasis on complications. Surgery. 2003, 134:647-53; discussion 654-5. 10.1016/s00396060(03)00312-x

12. Lesurtel M, Cherqui D, Laurent A, Tayar C, Fagniez PL: Laparoscopic versus open left lateral hepatic lobectomy: a case-control study. J Am Coll Surg. 2003, 196:236-42. 10.1016/S1072-7515(02)01622-8

13. Buell JF, Thomas MJ, Doty TC, et al.: An initial experience and evolution of laparoscopic hepatic resectional surgery. Surgery. 2004, 136:804-11. 10.1016/i.surg.2004.07.002

14. Morino M, Morra I, Rosso E, Miglietta C, Garrone C: Laparoscopic vs open hepatic resection: a comparative study. Surg Endosc. 2003, 17:1914-8. 10.1007/s00464-003-9070-4

15. Koffron AJ, Auffenberg G, Kung R, Abecassis M: Evaluation of 300 minimally invasive liver resections at a single institution: less is more. Ann Surg. 2007, 246:385-92; discussion 392-4. 10.1097/SLA.0b013e318146996c

16. Aldrighetti L, Pulitanò C, Catena M, et al.: A prospective evaluation of laparoscopic versus open left lateral hepatic sectionectomy. J Gastrointest Surg. 2008, 12:457-62. 10.1007/s11605-007-0244-6

17. Polignano FM, Quyn AJ, de Figueiredo RS, Henderson NA, Kulli C, Tait IS: Laparoscopic versus open liver segmentectomy: prospective, case-matched, intention-to-treat analysis of clinical outcomes and cost effectiveness. Surg Endosc. 2008, 22:2564-70. 10.1007/s00464-008-0110-y

18. Slakey DP, Simms E, Drew B, Yazdi F, Roberts B: Complications of liver resection: laparoscopic versus open procedures. JSLS. 2013, 17:46-55. 10.4293/108680812X13517013317716

19. Qiu J, Chen S, Pankaj P, Wu H: Laparoscopic hepatectomy is associated with considerably less morbidity and a long-term survival similar to that of the open procedure in patients with hepatic colorectal metastases. Surg Laparosc Endosc Percutan Tech. 2014, 24:517-22. 10.1097/SLE.0b013e31829cec2b 


\section{Cureus}

20. Nassar MN, El-Shakhs SA, M Sultan HM, El-Sisy AA, Sultan TA, Hafez SA: Short-term outcome after laparoscopic versus open liver resection in liver tumors. Menoufia Med J. 2015, 28:818-26.

21. Lau B, Franken C, Lee D, Putchakayla K, DiFronzo LA: Short-term outcomes of laparoscopic versus open formal anatomical hepatectomy: a case matched control study. Am Surg. 2015, 81:1097-100. 10.1177/000313481508101037

22. Hu, B. S: Comparison of laparoscopic vs open liver lobectomy (segmentectomy) for hepatocellular carcinoma. World Journal of Gastroenterology. 2011, 17:4725-10. 10.3748/wjg.v17.i42.4725

23. Bryant R, Laurent A, Tayar C, Cherqui D: Laparoscopic liver resection-understanding its role in current practice: the Henri Mondor Hospital experience. Ann Surg. 2009, 250:103-11. 10.1097/SLA.0b013e3181ad6660

24. Laurent A, Cherqui D, Lesurtel M, Brunetti F, Tayar C, Fagniez PL: Laparoscopic liver resection for subcapsular hepatocellular carcinoma complicating chronic liver disease. Arch Surg. 2003, 138:763-9; discussion 769. 10.1001/archsurg.138.7.763

25. Gagner M, Rheault M, Dubuc J: Laparoscopic partial hepatectomy for liver tumor. Surg Endoscopy. 2004, 6:97-8. 